\title{
Sound Attenuation Study of Micro-Scale Acoustic Package
}

\author{
Fuxi Zhang \\ College of Science and Engineering, Shanghai Ocean University, Shanghai Ocean Renewable Energy Research \\ Center, 999 Huchenghuan Road, Shanghai, 201306, China. \\ Department of Mechanical Engineering, Auburn University, 354 War Eagle Way, Auburn University, AL, 36849, \\ USA.
}

\section{George T. Flowers}

Department of Mechanical Engineering, Auburn University, 3428 Wiggins Hall, Auburn University, AL, 36849, USA.

\section{Robert N. Dean}

Department of Electrical and Computer Engineering, Auburn University, 222 Broun Hall, Auburn University, AL, 36849, USA.

\section{Jeffrey C. Suhling}

Department of Mechanical Engineering, Auburn University, 1418G Wiggins Hall, Auburn University, AL, 36849, USA.

\section{Jordan C. Roberts \\ Department of Mechanical Engineering, Auburn University, 2452 Wiggins Hall, Auburn University, AL, 36849, USA.}

(Received 3 November 2017; accepted 26 January 2018)

Certain microelectromechanical systems (MEMS), particularly MEMS gyroscopes, are notably susceptible to high power acoustic noise, especially when the noise is at or near its resonant frequency. A micro scale open-through dual expansion chamber (ODEC) array package with a continuous transmission loss (TL) in a wide frequency range is proposed to diminish the impact of such noise on the performance of MEMS gyroscopes. An analytical model based on planar wave propagation in stationary air has been developed with the consideration of the thermoacoustic effect near the inner rigid boundary regarding small scale. Experiments with ODEC groups and control groups (non-ODEC) samples have been conducted to verify the model and compare the performances. The ODECs perform in the manner of low-pass filters and both the experimental and the analytical results exhibit greater TL in the higher frequency ranges as compared to the corresponding control samples. In addition, the resonance effects of the ODEC itself are also experimentally observed to be a key factor in influencing the TL.

\section{NOMENCLATURE}

$r_{t}$ and $r_{c} \quad$ radii of tubes and chambers

$l_{t}$ and $l_{c} \quad$ length of tubes and chambers

$\Delta l \quad$ Length

$S \quad$ cross section area of tube or chamber

$p \quad$ acoustic pressure

$v \quad$ volume velocity

$U \quad$ particle velocity

$Y \quad$ characteristic impedance

$k \quad$ wave number

$\omega \quad$ angular frequency

$c \quad$ speed of sound in air

$\rho \quad$ density of air

$\delta_{v} \quad$ acoustic boundary layer thickness

$\mu \quad$ coefficient of dynamic viscosity

$\mu_{e} \quad$ effective coefficient of viscothermal friction $v_{k} \quad$ kinematic viscosity

$\gamma \quad$ heat capacity ratio

$C_{p}$ heat capacity

$C_{v} \quad$ specific heat

$K$ thermal conductivity

$f \quad$ viscous force

\section{INTRODUCTION}

Harsh environments are a major cause of the malfunction of compact electronic devices. Micro-scale MEMS gyroscopes, utilizing extremely small proof masses, are notably susceptible to the resonance caused by incident high power acoustic noise. Dean et al. showed that MEMS gyroscopes have unreliable performance when the acoustic noise levels approach 120 to $140 \mathrm{~dB}$ (ref: $20 \mu \mathrm{Pa}$ ) near the resonant frequency range of the sample devices. ${ }^{1,2}$ Thermal effects are also as a major cause 
of deterioration in electronic devices. ${ }^{3,4}$ It is quite challenging to design a packaging structure achieving good acoustic attenuation, as well as good thermal characteristics for an enclosed device. ${ }^{5-7}$ An open-through rigid substructure is considered as one possible strategy to meet such specifications.

Considerable work has been performed in the specific area of acoustic attenuation. Yunker and Flowers offered one approach by applying micro scale Helmholtz resonator arrays to a silicon-based acoustic metamaterial, which achieved a notch filter with an attenuation of $18 \mathrm{~dB}$ at $14.5 \mathrm{kHz}$ over a $700 \mathrm{~Hz}$ narrow band. ${ }^{6}$ Soobramaney proposed an approach of vibroacoustic isolating porous pads with an average $65 \%$ acoustic absorption at around $15 \mathrm{kHz}$ over a wide band. ${ }^{7}$ Selamet's derivation showed that a single expansion chamber system (in large dimension) produced a transmission loss with a repeating dome-dip pattern within a frequency range from 0 to $6 \mathrm{kHz}{ }^{8-10}$ Gerges et al, Munjal et al, and Kanade obtained similar conclusions to those of Selamet. ${ }^{11-13}$

However, there is considerable need for attenuation over a broad frequency band, instead of periodic dome-dip attenuation patterns, to adequately protect a specific MEMS device for which the resonant frequency may be quite different to that of the nominal design frequency. In addition, the isolation package and inner structure are required to be in very small dimensions and a through-hole structure for convection is desired. With regard to the small dimensions, Munjal, Antao et al, Friend et al, and Karlsen et al provided studies of the thermoacoustic effect at rigid boundaries (or walls) causing additional transmission losses. ${ }^{14-17}$ Inspired by these works, this current study proposes a further extension of a micro scale open-through dual expansion chamber (ODEC) package by employing planar wave propagation in stationary air and the thermo-acoustic effect to achieve high and continuous attenuations in a wide frequency range. Corresponding experiments with several configurations of the ODECs and related control group samples (non-ODEC) were conducted to validate the theoretical predictions with respect to transmission loss (TL) already defined in abstract). The geometric effect of the TL is also investigated with respect to chamber length and radius.

\section{THEORETICAL APPROACHES}

The concentric configuration of a single element ODEC for plane wave propagation is illustrated in Fig. 1. It consists of three circular tubes with radius, $r_{t}$, and length, $l_{t}$, and two circular chambers with radius, $r_{c}$, and length, $l_{c}$. The areas with section lines represent the solid rigid structures, and the blank area represents the fluid medium, air.

As illustrated in Fig. 1, the incident plane wave at the entrance of the ODEC element can be described by the acoustic pressure $p_{i}=A_{i}+B_{i}$ and the volume velocity $v_{i}=$ $\frac{1}{Y_{x}}\left(A_{i}-B_{i}\right)=u_{i} \rho_{0} S$, where $u_{i}$ is the particle velocity associated with $p_{i}$ governed by the wave equation in Eq. (1).

$$
\frac{\partial^{2} p}{\partial x^{2}}=\frac{1}{c^{2}} \frac{\partial^{2} p}{\partial t^{2}}
$$

The parameters $\rho_{0}$ and $S$ represent the density of air at $20^{\circ} \mathrm{C}$ and the cross-section area of tube or chamber. The acoustic pressure and volume velocity of the downstream $(x>0)$ can be expressed as follows:

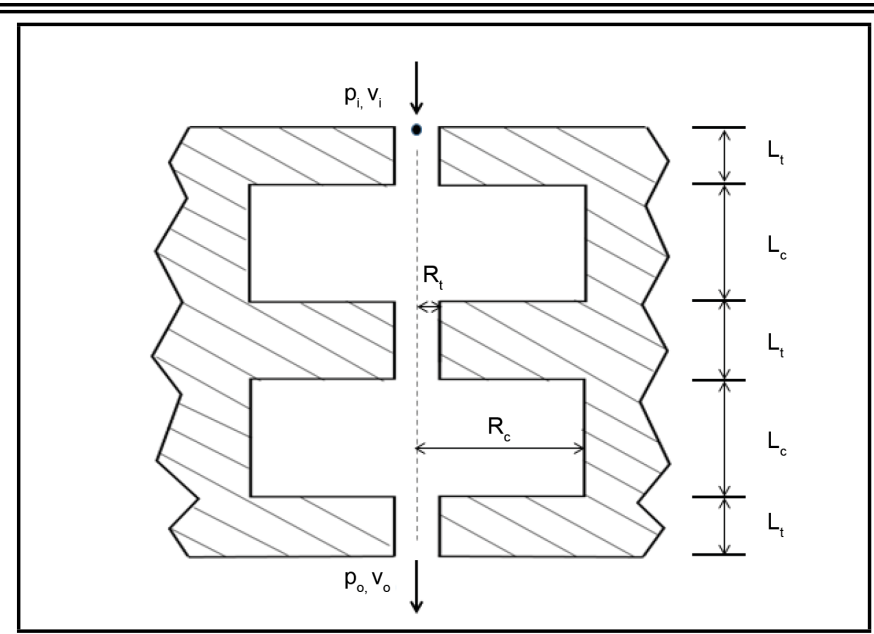

Figure 1a. Cross section view in 2-D and configuration of the ODC element.

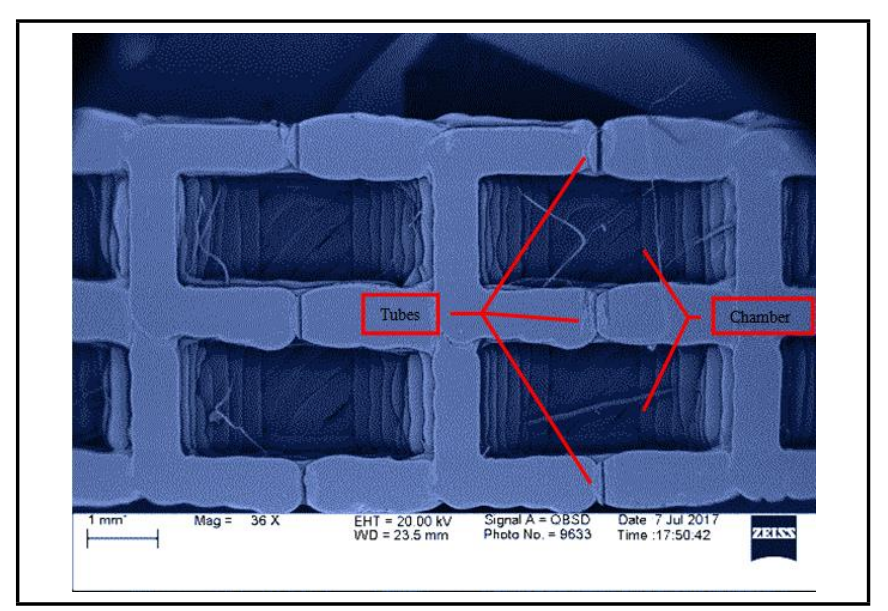

Figure 1b. Magnified cross section picture of the ODC array, corresponding to (a).

$$
\begin{aligned}
p_{x}= & A_{i} e^{-j k \widetilde{l}_{t}}+B_{i} e^{+j k \widetilde{l}_{t}}=\left(A_{i}+B_{i}\right) \cos k \widetilde{l}_{t}- \\
& j\left(A_{i}-B_{i}\right) \operatorname{sink} \widetilde{l}_{t}=p_{i} \cos k \widetilde{l}_{t}-j Y_{x} v_{i} \operatorname{sink} \widetilde{l}_{t}
\end{aligned}
$$

$$
\begin{gathered}
v_{x}=\frac{A_{i} e^{-j k \widetilde{l}_{t}}-B_{i} e^{+j k \widetilde{l}_{t}}}{Y_{x}}=\frac{\left(A_{i}-B_{i}\right)}{Y_{x} \cos \widetilde{l}_{t}}-\frac{j\left(A_{i}+B_{i}\right)}{Y_{x} \operatorname{sink} \widetilde{l}_{t}}= \\
v_{i} \operatorname{cosk} \widetilde{l}_{t}-j p_{i} / Y_{x} \operatorname{sink} \widetilde{l}_{t} \\
\widetilde{l_{t}}=\Delta l+l_{t}
\end{gathered}
$$

$k=\frac{\omega}{c}$ refers to the wave number with respect to the wavelength $\lambda$. Parameter $Y_{x}=\frac{c}{S}$ represents the characteristic impedance of a cross section of $S$ at the distance $x=0 . l_{t}$ and $\Delta l=\frac{8 r}{3 \pi}$ refer to the tube length and the corresponding length correction shown in Eq. (4).

By applying Eq. (2) to Eq. (4), the correlation between the acoustic pressure and volume velocity via the inlet and downstream $(x>0)$ can be expressed in the following transfer matrix equation:

$$
\left[\begin{array}{c}
p_{i} \\
v_{i}
\end{array}\right]=\left[\begin{array}{cc}
\operatorname{cosk} \widetilde{l}_{t} & j Y_{x} \operatorname{sink} \widetilde{l_{t}} \\
j / Y_{x} \operatorname{sink} \widetilde{l_{t}} & \operatorname{cosk} \widetilde{l}_{t}
\end{array}\right]\left[\begin{array}{l}
p_{x} \\
v_{x}
\end{array}\right] .
$$


Table 1. The air properties at constant temperature. ${ }^{21}$

\begin{tabular}{||c|c|c|c|c|c|c||}
\hline $\begin{array}{c}T \\
{\left[{ }^{\circ} \mathrm{C}\right]}\end{array}$ & $\begin{array}{c}\rho \\
{\left[\mathrm{kg} / \mathrm{m}^{3}\right]}\end{array}$ & $\begin{array}{c}v_{k} \\
{\left[\times 10^{-6}\right.} \\
\left.\mathrm{m}^{2} / \mathrm{s}\right]\end{array}$ & $P_{r}$ & $\mathrm{~K}$ & $\mathrm{c}$ & $\gamma$ \\
\hline 20 & 1.205 & 15.11 & 0.713 & 0.0257 & 343.28 & 1.401 \\
\hline
\end{tabular}

By repeating Eq. (5) and employing the length of chamber $l_{c}$, it can easily get the correlation between the inlet and the outlet as follows:

$$
\left[\begin{array}{c}
p_{i} \\
v_{i}
\end{array}\right]=\prod_{0}^{x}[M]\left[\begin{array}{l}
p_{o} \\
v_{o}
\end{array}\right]=\left[\begin{array}{ll}
T_{11} & T_{12} \\
T_{21} & T_{22}
\end{array}\right]\left[\begin{array}{l}
p_{o} \\
v_{o}
\end{array}\right]
$$

where $[M]$ refers to the transfer matrix in Eq. (5).

Due to the micro-scale of the ODEC structure (under the radial distance threshold: $1.25 \times \delta_{v}=1.25 \times \sqrt{\frac{2 \mu}{\rho_{0} \omega}}$, and $\delta_{v}$ known as the acoustic boundary layer thickness), the particle velocity $u_{x}$ decreases from the maximum in the center to zero at the rigid wall by the viscous force $f=\frac{\mu S\left(\partial u_{x}\right)}{\partial r}$, and consequently causes additional absorption. Here $\mu=v_{k} \rho$ is the coefficient of dynamic viscosity of the medium. $v_{k}$ and $\rho$ represent the kinematic viscosity and ambient density of air, respectively. $\mu$ is a function of temperature but independent of frequency. The characteristic impedance $Y_{x}$ can be expressed as a function correlated with $\mu$ to realize the viscous absorption in the duct system, ${ }^{19,20}$

$$
Y_{x}=\frac{p_{i}}{v_{i}}= \pm \frac{v_{i}}{\pi r^{2}}\left\{1-\frac{1}{r}\left(\frac{\mu_{e}}{2 \rho_{0} \omega}\right)^{\frac{1}{2}}+\frac{j}{r}\left(\frac{\mu_{e}}{2 \rho_{0} \omega}\right)^{\frac{1}{2}}\right\}
$$

where $r$ is the radius of each section (such as the tube radii $R_{t}$ and chamber radii $R_{c}$ ), and the minimum $r$ is set to $45 \mu \mathrm{m}$ to allow $Y_{x}$ to be physically meaningful. $\mu_{e}$ is the effective coefficient of viscothermal friction as described in Eq. (8):

$$
\mu_{e}=\mu\left[1+\left(\gamma^{\frac{1}{2}}-\frac{1}{\gamma^{\frac{1}{2}}}\right)\left(\frac{K}{\mu C_{p}}\right)^{\frac{1}{2}}\right]^{2} ;
$$

where $\gamma=\frac{c_{p}}{c_{v}}$ is the heat capacity ratio. The parameter $K$ is the thermal conductivity. $C_{p}$ is the heat capacity at constant pressure. $C_{v}$ is the specific heat in a constant volume process. The air properties used in this analysis are listed in Table 1.

Considering the magnitude of the plane wave acoustic pressure at both open ends of the ODEC structure, and assuming there is no reflection at the outlet end, which results in $p_{o}=A_{o}$ and $p_{i}=2 A_{i}-Y_{x} v_{i}$. This magnitude can be expressed in terms of elements in the transfer matrix $[T]$ :

$$
\begin{aligned}
& A_{i}=\frac{p_{i}+Y_{x} v_{i}}{2}= \\
& {\left[\left(T_{11} A_{o}+T_{12} \frac{A_{o}}{Y_{o}}\right)+\frac{\left(T_{21} A_{o}+T_{22} \frac{A_{o}}{Y_{o}}\right)}{2}\right] .}
\end{aligned}
$$

Consequently, the TL can be determined as:

$$
\begin{aligned}
& \mathrm{TL}=20 \log _{10}\left|\frac{A_{i}}{A_{o}}\right|=20 \log _{10} \\
& {\left[\left(\frac{Y_{o}}{Y_{x}}\right)^{0.5}\left|\frac{1}{2}\left(T_{11}+\frac{T_{12}}{Y_{o}}+Y_{x} T_{21}+\frac{Y_{x}}{Y_{o}} T_{22}\right)\right|\right] .}
\end{aligned}
$$

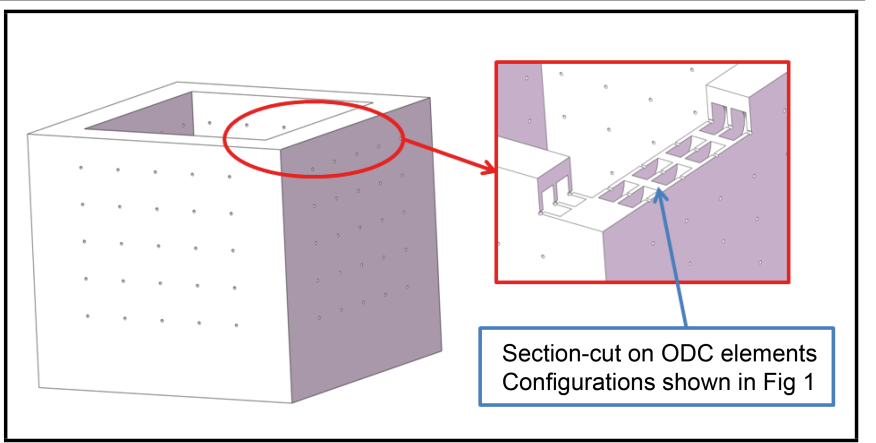

Figure 2a. 3-D schematic of the metamaterial package design.

Reorganizing Eq. (10) by substituting Eqs. (6), (7) and (8) into it, the terms $T_{i j}$ become a quintic function of sines multiplying $Y_{x}$ (real for $i=j$ and imaginary for $i \neg j$ ). Therefore, the absolute value in Eq. (10) increases from 0 in the manner of non-linear curve with increasing frequency $\omega$.

\section{EXPERIMENTAL SAMPLES AND TESTING SETUP}

All samples were designed in the form of a one-side-open box with the same inner dimensions of a $25.4 \mathrm{~mm}$ cube. Wall thicknesses vary from 4.5 to $5 \mathrm{~mm}$ with $0.5 \mathrm{~mm}$ increment, which is correlated with the increasing chamber length $l_{c}$. The ODEC elements array, of which the tube and chamber configurations were listed in Table 2, was fabricated through the walls of each ODEC group sample. The corresponding control samples were comprised of solid walls with the same thickness as the corresponding ODEC group sample. All the ODEC group and control samples were fabricated with the same material, as illustrated in Fig. 2.

A series of experiments were conducted in the Acoustics Laboratory at Auburn University. In order to obtain the sound level measurements, an omnidirectional microphone was firmly encased by a test sample and an isolation case. The incident sound was projected perpendicularly to the side wall of the test samples with a $105 \mathrm{~dB}$ SPL (ref: $20 \mu \mathrm{Pa}$ ) and frequency range from $0 \mathrm{~Hz}$ to $8200 \mathrm{~Hz}$. Fig. 2c shows a schematic diagram of the experimental setup used in the acoustic testing. Due to the minute structure of the ODEC elements, variations resulting from the sample fabrication process and test setup implementation may yield unexpected outcomes. So, a series of vibration tests were conducted as well to determine each sample's natural frequencies, which was utilized as a reference factor in analyzing the experimental results.

\section{RESULTS AND DISCUSSION}

The theoretical model (Eq. (10)) was used to calculate a variety of transmission loss predictions for Group 1 and 2, illustrated in Fig. 3a, which were validated by the corresponding experiments. Fig. 4a shows comparisons between the model predictions and experimental results for the ODEC samples and the control samples.

The predicted TL of Group 1 sample 1 continuously increases over the frequency range $0 \mathrm{~Hz}$ to $8200 \mathrm{~Hz}$ with some mild variations. A local maximum of $22.38 \mathrm{~dB}$ occurs at $2800 \mathrm{~Hz}$ where there is a mildly convex appearance to the 


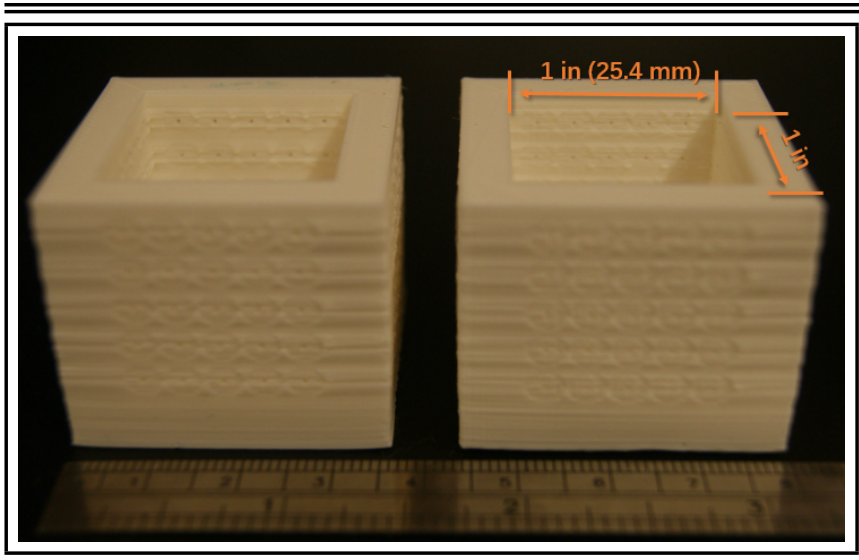

Figure 2b. Photo of fabricated testing samples.

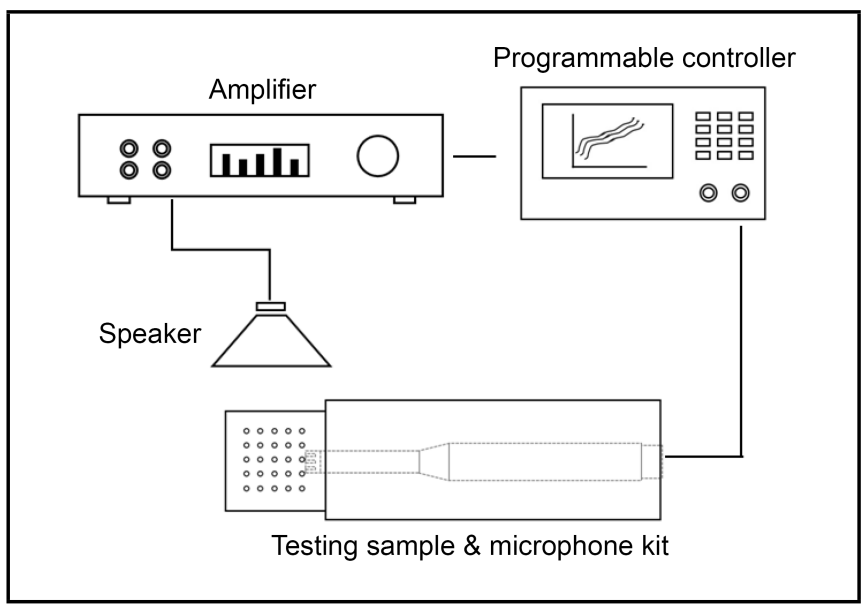

Figure 2c. The schematic of testing equipment with customized isolation case.

curve, and a local minimum of $20.25 \mathrm{~dB}$ occurs at $4100 \mathrm{~Hz}$ where there is a mildly concave appearance. The highest TL value is $45.25 \mathrm{~dB}$ at $8200 \mathrm{~Hz}$, which is at highest frequency considered in this analysis. Similarly, convex and concave characteristics can be observed in all the prediction results for Group 1 and 2. The corresponding local maxima, minima, and highest TL values are listed in Table 2. As observed, the predicted behavior of the micro-scale ODEC are in the form of a continuous low pass filter instead of the periodic dome-dip attenuation performance, which differs dramatically from the behavior observed for chamber systems in large scale. ${ }^{8-13}$

Six groups of ODEC boxes were designed, fabricated and tested to validate and compare the analytical predictions. For comparison purposes, the three samples in each group share the same total wall thickness but differ in the radii of the expansion chambers. Table 2 summarizes the specifics of the detailed geometric configurations and the experimental results of the ODEC metamaterial groups. The lengths of the expansion chambers are reduced by $250 \mu \mathrm{m}$ for each group, which serves to decrease the total thickness of the walls. The chamber radii are held constant between Groups 1 to 3 and between Groups 4 to 6 .

Fig. 4a shows a comparison between the experimentally measured TL values for the control groups and the ODEC groups. Overall, the experimental results for all ODEC groups correlate well with their corresponding analytical predictions within the same frequency band. The experimental results for

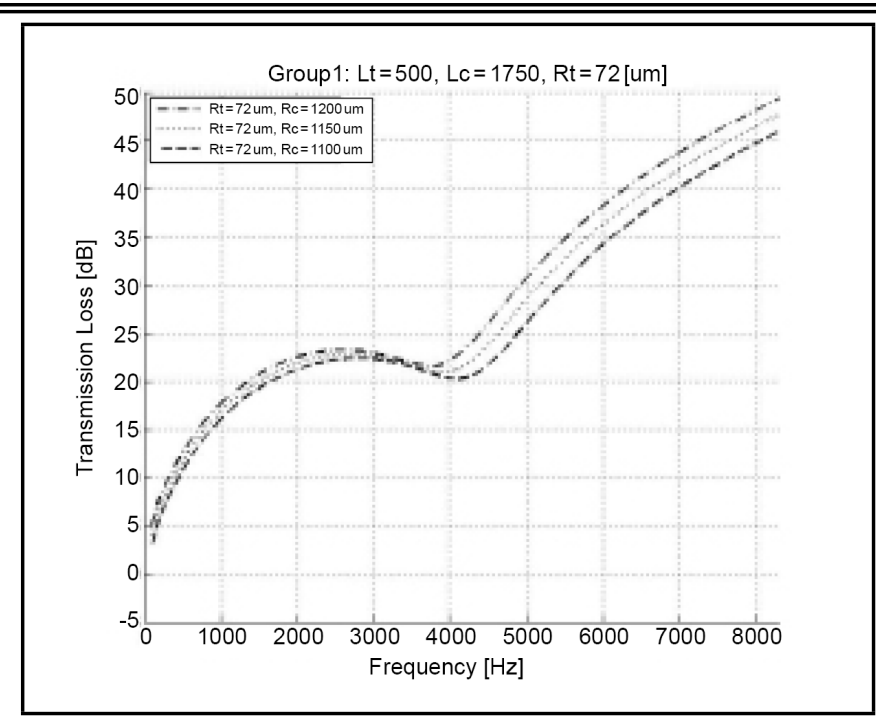

Figure 3a. Analytical predictions. Group 1, total element length: $5 \mathrm{~mm}$.

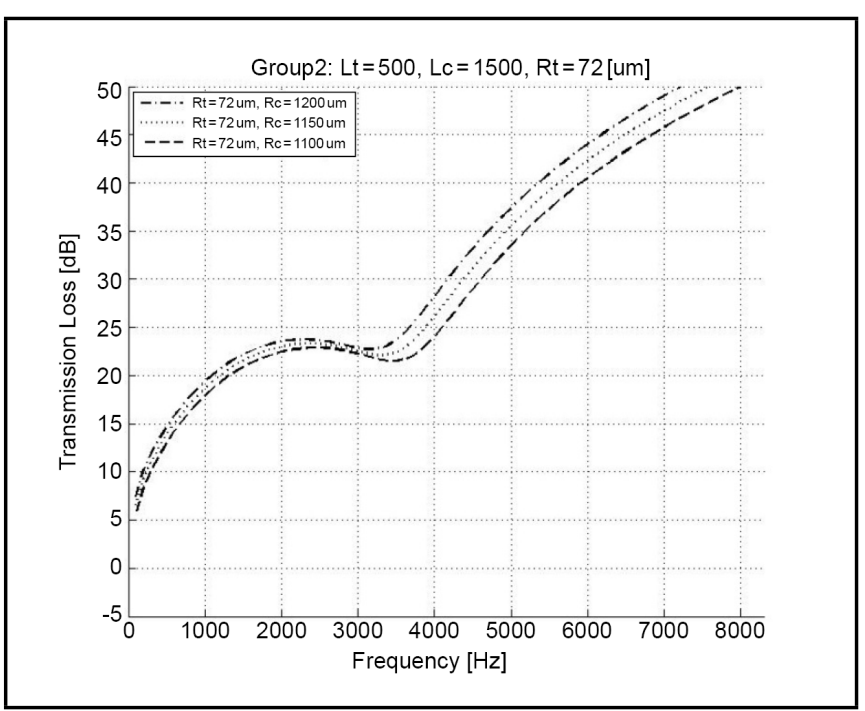

Figure 3b. Analytical predictions. Group 2, total element length: $4.5 \mathrm{~mm}$.

the control group samples exhibited somewhat weaker performance than the corresponding analytical predictions. The attenuation performance of the control samples is highly dependent on the material damping characteristics, which are probably not perfectly captured by the linear model assumed in the analysis. This may explain the observed differences. In contrast to the control groups, all six groups of ODEC achieve correspondingly better attenuation performance except in the frequencies below the intersection area $(\mathrm{E})$. This may be due to the level of precision in the sample fabrication, as well as modeling inaccuracies.

In addition, some of the observed differences may be due to the un-modeled dynamics of the test structure. Further experiments were conducted to further evaluate these effects and are discussed in Section 4.3.

\subsection{Comparison of Control Groups to ODEC Metamaterial Groups}

In order to facilitate discussion of the experimentally observed behavior in Fig. 4a, several key sections of the respec- 
Table 2. Detailed geometry configuration and testing results of these ODEC groups.

\begin{tabular}{|c|c|c|c|c|c|c|c|c|c|c|}
\hline & $\begin{array}{c}\text { Sample } \\
\text { No. }\end{array}$ & $r_{t}[\mu \mathrm{m}]$ & $r_{c}[\mu \mathrm{m}]$ & $l_{t}[\mu \mathrm{m}]$ & $l_{c}[\mu \mathrm{m}]$ & $\xi$ & $\begin{array}{l}\text { Approximate } \\
\text { frequency of } \\
\text { 1st concave } \\
\text { section }[\mathrm{Hz}]\end{array}$ & $\begin{array}{l}\text { Approximate } \\
\text { frequency of } \\
\text { convex } \\
\text { section }[\mathrm{Hz}]\end{array}$ & $\begin{array}{l}\text { Approximate } \\
\text { frequency of } \\
\text { 2nd concave } \\
\text { section }[\mathrm{Hz}]\end{array}$ & $\begin{array}{c}\mathrm{TL} \text { at } \\
8.1 \mathrm{kHz}[\mathrm{dB}]\end{array}$ \\
\hline \multirow[t]{3}{*}{ Group 1} & 1 & \multirow[t]{3}{*}{72} & 1100 & \multirow[t]{3}{*}{500} & \multirow[t]{3}{*}{1750} & 816.94 & \multirow[t]{3}{*}{640} & \multirow[t]{3}{*}{5315} & \multirow[t]{3}{*}{6451} & 39.60 \\
\hline & 2 & & 1150 & & & 892.89 & & & & 44.15 \\
\hline & 3 & & 1200 & & & 972.22 & & & & 44.51 \\
\hline \multirow[t]{3}{*}{ Group 2} & 1 & \multirow[t]{3}{*}{72} & 1100 & \multirow[t]{3}{*}{500} & \multirow[t]{3}{*}{1500} & 700.23 & \multirow[t]{3}{*}{604} & \multirow[t]{3}{*}{5412} & \multirow[t]{3}{*}{6834} & 36.85 \\
\hline & 2 & & 1150 & & & 765.33 & & & & 35.82 \\
\hline & 3 & & 1200 & & & 833.34 & & & & $\begin{array}{l}38.38 \\
\end{array}$ \\
\hline \multirow[t]{3}{*}{ Group 3} & 1 & \multirow[t]{3}{*}{72} & 1100 & \multirow[t]{3}{*}{500} & \multirow[t]{3}{*}{1250} & 583.53 & \multirow[t]{3}{*}{570} & \multirow[t]{3}{*}{5097} & \multirow[t]{3}{*}{6481} & 30.87 \\
\hline & 2 & & 1150 & & & 637.78 & & & & 32.09 \\
\hline & 3 & & 1200 & & & 694.45 & & & & 33.20 \\
\hline \multirow[t]{3}{*}{ Group 4} & 1 & \multirow[t]{3}{*}{45} & 900 & \multirow[t]{3}{*}{500} & \multirow[t]{3}{*}{1000} & 800.00 & \multirow[t]{3}{*}{570} & \multirow[t]{3}{*}{4499} & \multirow[t]{3}{*}{6416} & 28.87 \\
\hline & 2 & & 950 & & & 891.36 & & & & 32.77 \\
\hline & 3 & & 1000 & & & 987.66 & & & & 31.05 \\
\hline \multirow[t]{3}{*}{ Group 5} & 1 & \multirow[t]{3}{*}{45} & 900 & \multirow[t]{3}{*}{500} & \multirow[t]{3}{*}{750} & 600.00 & \multirow[t]{3}{*}{538} & \multirow[t]{3}{*}{4219} & 6100 & 29.52 \\
\hline & 2 & & 950 & & & 668.52 & & & & 35.27 \\
\hline & 3 & & 1000 & & & 740.75 & & & & 32.85 \\
\hline Group 6 & 1 & 45 & 900 & 500 & 500 & 400.00 & 508 & 3816 & 5424 & 31.32 \\
\hline & 2 & & 950 & & & 445.68 & & & & 37.16 \\
\hline & 3 & & 1000 & & & 493.83 & & & & 33.98 \\
\hline
\end{tabular}

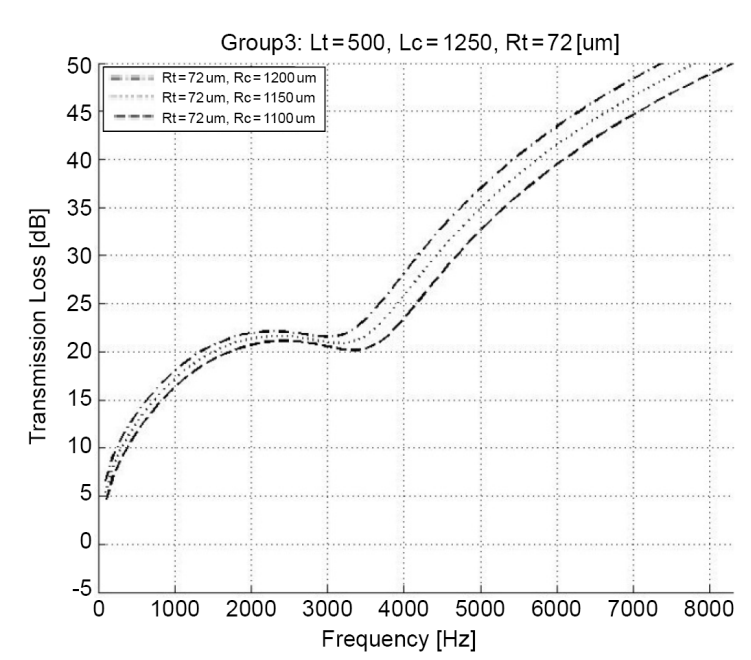

Figure 3c. Analytical predictions. Group 3, total element length: $4 \mathrm{~mm}$.

tive curves are denoted by labels, as indicated in the following descriptions. "A" denotes the first concave section of the ODEC and control samples. "B" denotes the second concave section of the ODEC and control samples. "C" denotes the largest peaked convex section of the ODEC samples. "D" denotes the largest peak of the control sample. "E" denotes the intersection region between the control and ODEC curves.

Inspection of the experimental results for the control samples of Group 1 shows a low peak in the starting frequency range (A), from 0 to $0.5 \mathrm{kHz}$ at around $10-12 \mathrm{~dB}$. The maximum TL for the Group 1 control samples (D) occurs at $1.2 \mathrm{kHz}$ with an attenuation of $37.61 \mathrm{~dB}$. For frequencies above that associated with the maximum TL, the TL decreases to $21.36 \mathrm{~dB}$ at approximately $6.5 \mathrm{kHz}$ before rising to approximately $25 \mathrm{~dB}$ at the end of the frequency range. Similar characteristics are observed for all five of the other control group samples, as well. After reaching the maximum attenuation level around $38 \mathrm{~dB}$

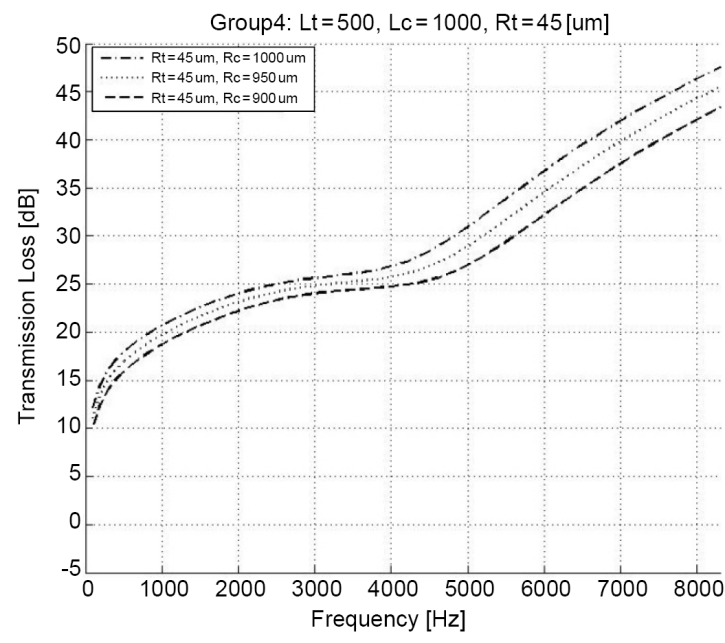

Figure 3d. Analytical predictions. Group 4, total element length: $3.5 \mathrm{~mm}$.

at about $1 \mathrm{kHz}$, the TL generally shows a sharply decreasing trend corresponding to the decreasing of wall thickness.

The experimental ODEC results largely agree with the corresponding analytical predictions over the entire frequency band. Unlike the control samples, the ODEC sample results showed similar transmission loss characteristics to those predicted by the model. However, there are several differences between the experimental results and the analytical predictions. The first is a low peak region (similar to that observed for the control samples but of lower amplitude) which has its highest TL at about $8 \mathrm{~dB}$ at the center frequency range of 0.5 to $0.6 \mathrm{kHz}$ and an adjacent concave section (A) with a minimum TL around $0.7 \mathrm{kHz}$. There is a second concave section (B) which show a minimum attenuation at the center frequency range of 5.4 to $6.8 \mathrm{kHz}(\mathrm{B})$. Between the two concave regions, there is a peaked convex section $(\mathrm{C})$, which also shows variation between the model prediction and the exper- 


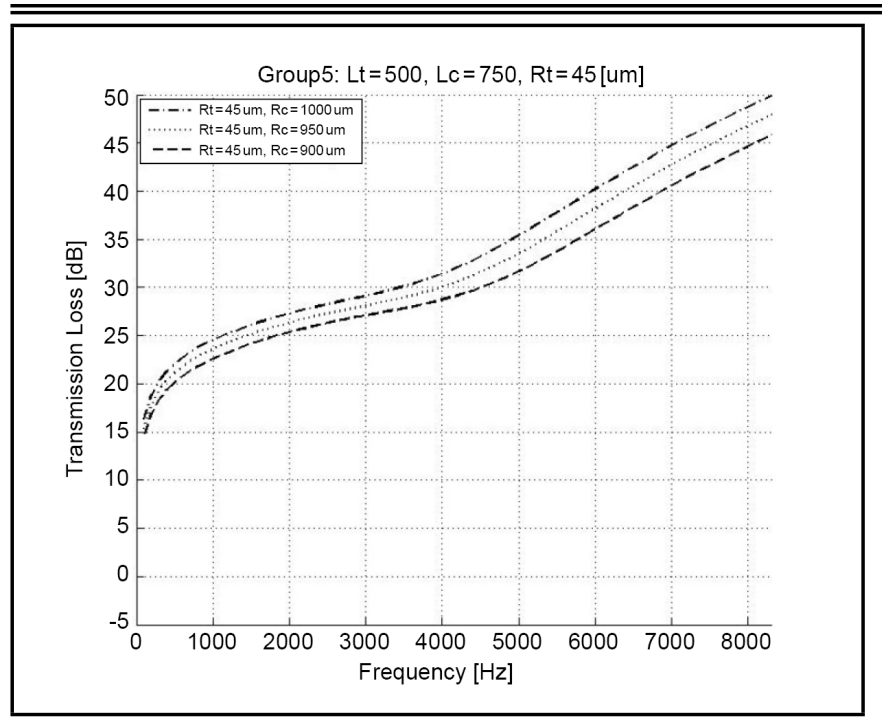

Figure 3e. Analytical predictions. Group 5, total element length: $3 \mathrm{~mm}$.

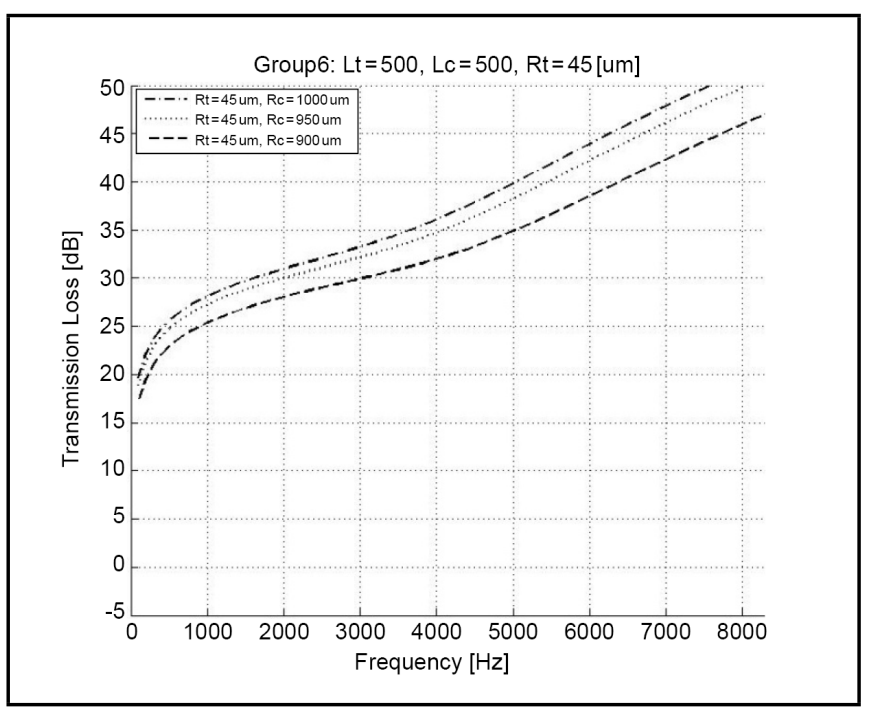

Figure 3f. Analytical predictions. Group 6, total element length: $2.5 \mathrm{~mm}$.

imentally observed results. The measured TL values for frequencies above the concave sections of the curves converged to the corresponding analytical results. All the metamaterial TL values are higher than those of the corresponding control groups after the frequencies for which the curves cross (E), for Group 1 to 6 , the values are $4.31 \mathrm{kHz}, 3.62 \mathrm{kHz}, 2.71 \mathrm{kHz}$, $2.42 \mathrm{kHz}, 2.28 \mathrm{kHz}$, and $2.03 \mathrm{kHz}$, respectively. The intersection frequencies decrease with the decreasing wall thicknesses of the control boxes. Table 2 and Fig. 4a summarize the results of these experiments. These results show that ODEC boxes have significantly higher transmission losses in these higher frequency ranges than do the corresponding control boxes. The TL differences between the ODEC and control boxes range from about 10 to $20 \mathrm{~dB}$, with Group 1 showing the best transmission loss performance.

\subsection{Effect of Geometric Configuration}

Another important design consideration is the overall effect of wall thickness on transmission loss. Accordingly, the six sample groups were designed with wall thicknesses starting at

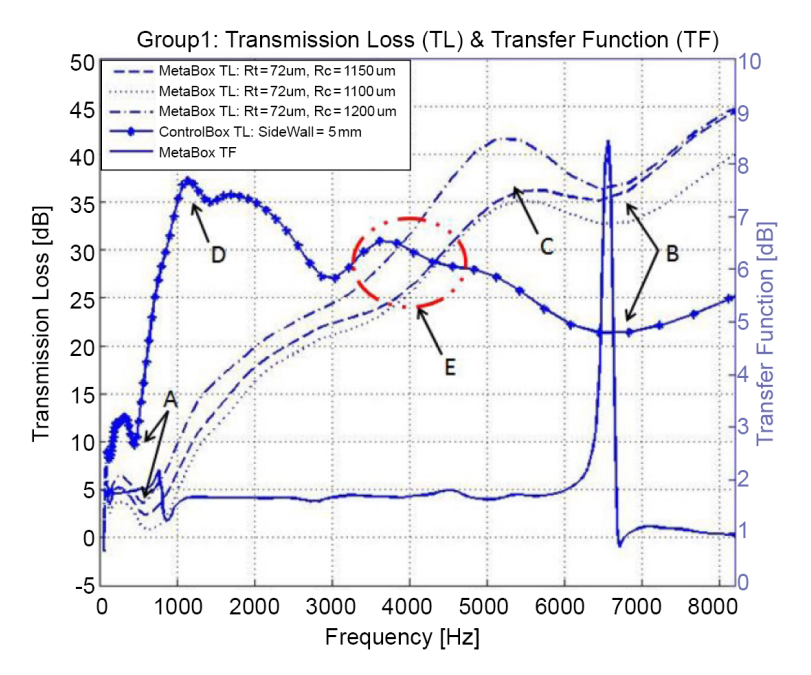

Figure 4a. Transmission loss from 0 to $8.2 \mathrm{kHz}$, Group 1 .

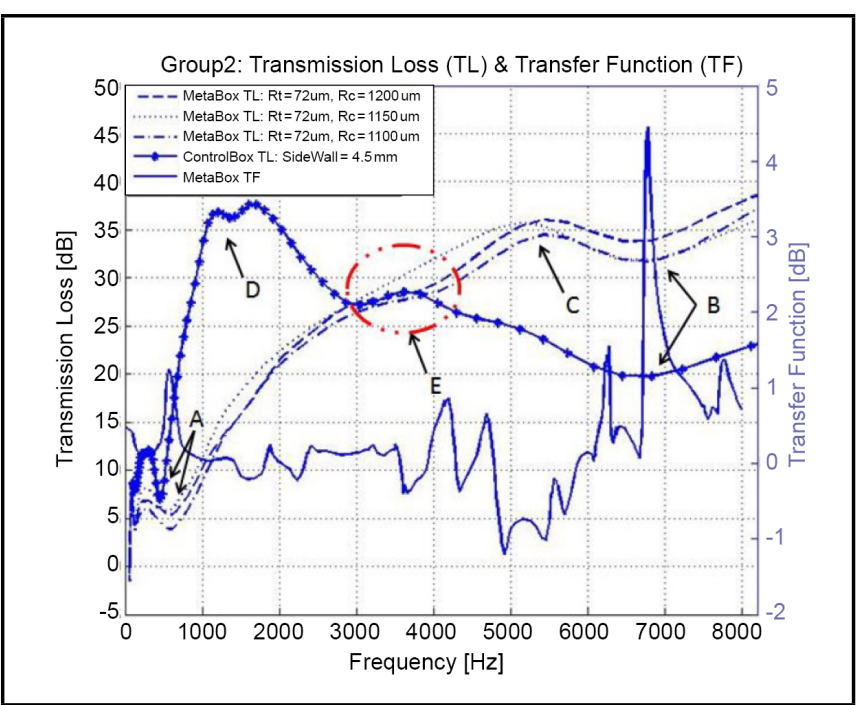

Figure 4b. Transmission loss from 0 to $8.2 \mathrm{kHz}$, Group 2 .

$5 \mathrm{~mm}$ for Group 1, with a reduction of $0.5 \mathrm{~mm}$ in each group down to a wall thickness of to $2.5 \mathrm{~mm}$ for Group 6. For the control groups, it can be observed from inspection of Fig. 4a that the transmission loss decreases significantly with wall thicknesses except in the low-frequency range. As was described in the previous section, for the purpose of consistency, the tube length of the ODEC samples was held constant at $500 \mu \mathrm{m}$. The length for each of the two chambers was set at a starting length of $1.75 \mathrm{~mm}$ for Group 1 and was reduced by $0.25 \mathrm{~mm}$ for each group down to Group 6, which resulted in the sample wall thicknesses starting at $5 \mathrm{~mm}$ and decreasing incrementally to $2.5 \mathrm{~mm}$.

The volume ratios, $\phi$, for each sample group are tabulated in Table 2. It can be observed by inspection of the analytical and experimental results from Fig. 3a and 4a, respectively, that the attenuation performance correlates to the volume ratio. For Groups 1 to 3, which share the same geometric configurations except for the chamber lengths, the TLs decrease in accordance with decreasing $\phi$ in the frequency range from the center frequency of the second concave section of each TL curve to the upper limit of the frequency range tested. A similar observation is also true for Groups 4 to 6 as well. Therefore, it appears 


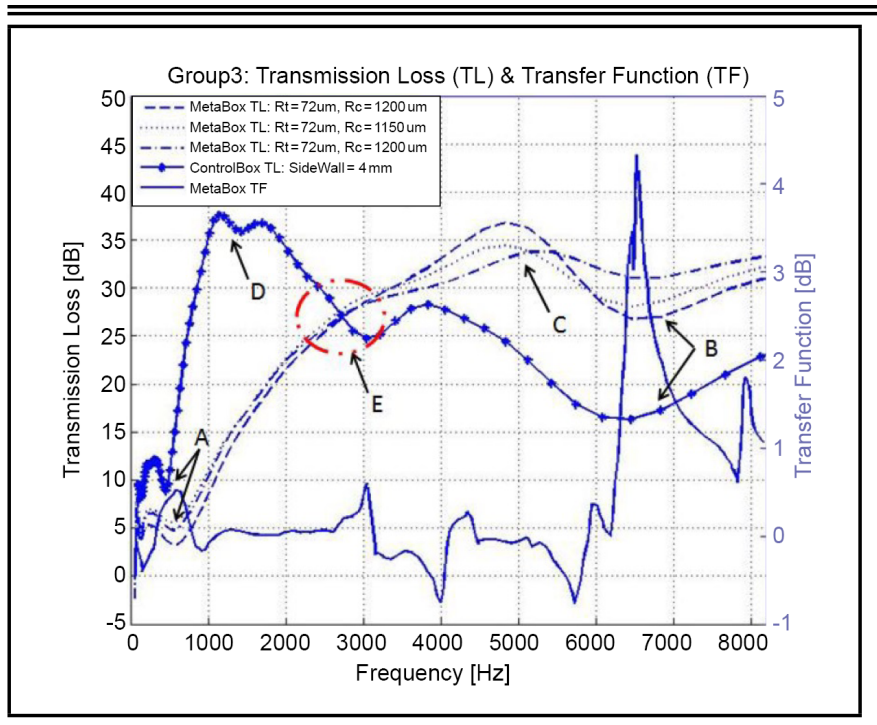

Figure 4c. Transmission loss from 0 to $8.2 \mathrm{kHz}$, Group 3 .

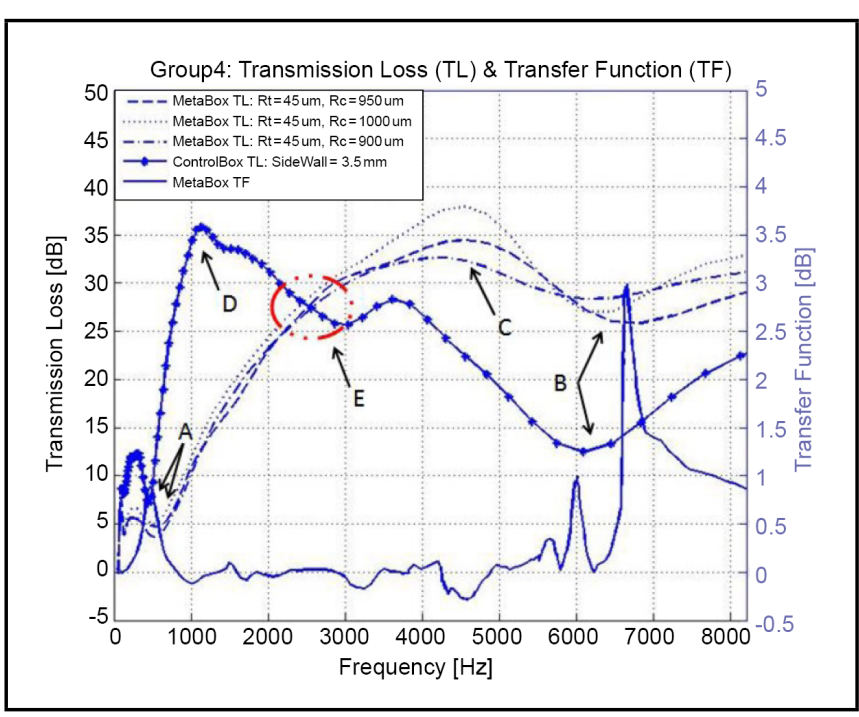

Figure 4d. Transmission loss from 0 to $8.2 \mathrm{kHz}$, Group 4 .

that volume ratio is a key parameter with regard to transmission loss, with a higher volume ratio generally producing a better attenuation performance.

\subsection{Vibration of the Metamaterial Structure}

As discussed in sections 4.1 and 4.2, there are some modest but clearly observable differences between the analytical predictions and the experimental results. These consist mainly of a convex bulge in the TL curves at moderate frequencies and concave sections that precede and follow. It is expected that these effects are related to the resonant characteristics of the gross structure of the sample boxes. In order to test this supposition, a series of vibration experiments were conducted using a vibratory shaker and a non-contacting laser displacement measurement system. The corresponding frequency responses of each group are illustrated in Fig. 4a, along with the associated transmission loss behavior.

Inspection of the resulting frequency responses, noted as "Metabox TF" curves in Fig.4, shows that there are two main peaks for each group. The first peaks appear in the frequency range from 0.5 to $0.8 \mathrm{kHz}$, with relatively small amplitudes. The second peaks, with larger amplitudes, appear in the fre-

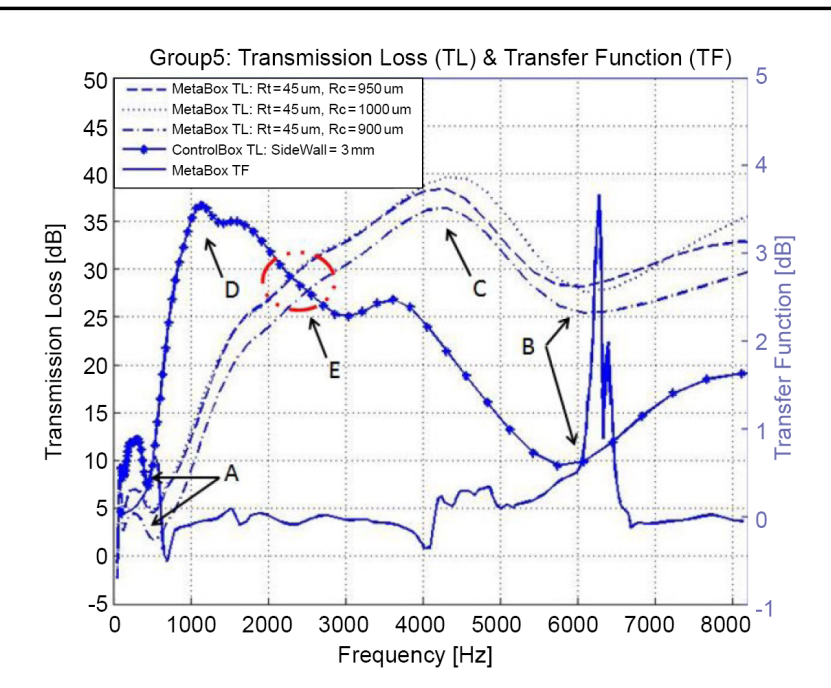

Figure 4e. Transmission loss from 0 to $8.2 \mathrm{kHz}$, Group 5 .

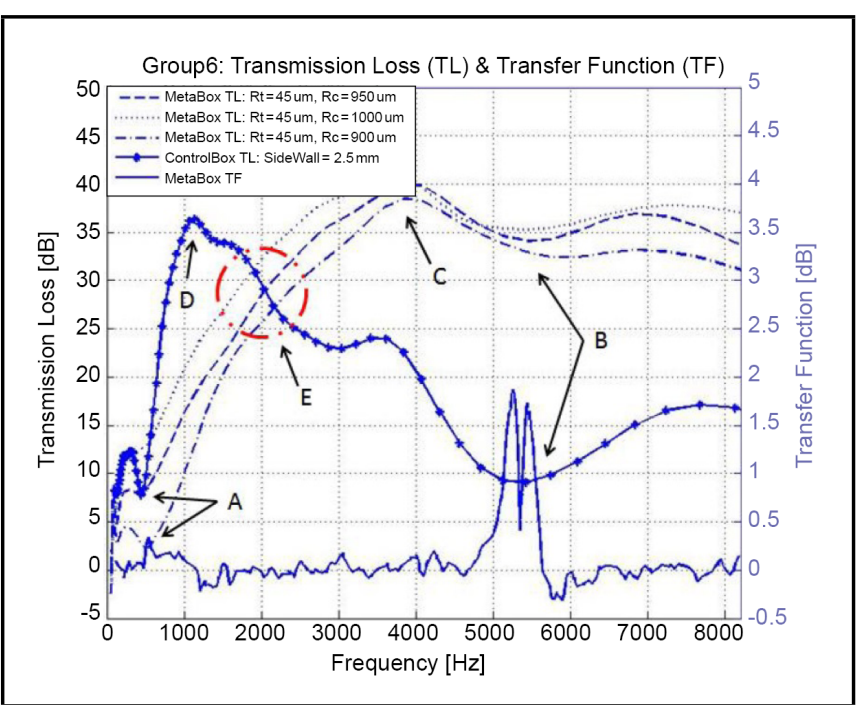

Figure 4f. Transmission loss from 0 to $8.2 \mathrm{kHz}$, Group 6 .

quency range from 5.3 to $6.8 \mathrm{kHz}$. As shown in Table 3 , both main resonant frequencies are almost identical to the frequencies associated with the first and second concave sections in each of the ODEC groups. Therefore, it appears that the concave sections of the TL curves (representing decreases in transmission loss) are associated with the first two resonant modes of the overall structure of the sample boxes. Likewise, the convex bulging section (representing increases in transmission loss) are associated with the corresponding anti-resonance points between the two resonant peaks.

\section{SUMMARY AND CONCLUSIONS}

A type of novel acoustic substructure consisting of a microscale open-through dual-expansion chamber (ODEC) element was devised with the objective of noise attenuation. By using an analytical approach which combined the Helmholtz equation and the thermoacoustic effect, an analytical model for a single element of the acoustic substructure was developed. Six groups of the ODEC and corresponding control groups were designed and fabricated. A series of experiments were conducted under the condition of a $105 \mathrm{~dB}$ noise incidence source 
Table 3. Natural frequencies of all ODEC groups [kHz].

\begin{tabular}{|c|c|c|c|c|c|c|}
\hline Natural Frequency & Group 1 & Group 2 & Group 3 & Group 4 & Group 5 & Group 6 \\
\hline $\mathrm{f}_{1}$ & 0.77 & 0.58 & 0.61 & 0.48 & 0.55 & 0.54 \\
\hline $\mathrm{f}_{2}$ & 6.56 & 6.78 & 6.53 & 6.66 & 6.27 & 5.34 \\
\hline
\end{tabular}

in a swept sine wave of from 0 to $8.2 \mathrm{kHz}$. The ODEC samples from all groups correlated well, but having some noted differences, with the overall transmission loss behavior observed from the corresponding analytical models. The ODEC groups were designed to provide improved noise attenuation in the upper-frequency range, as compared to the non-ODEC control boxes, and this result was clearly demonstrated by the experimental results. The ratio of the chamber volume to the tube volume was observed to have a strong influence on attenuation performance, with higher volume ratios, $\phi$, producing increaed transmission loss.

\section{REFERENCES}

1 Dean, R. N., Flowers, G. T., Hodel, A. S., Roth, G., Castro, S., Zhou, R., Moreira, A., Ahmed, A., Rifki, R., Grantham, B. E., Bittle, D., and Brunsch, J. On the degradation of MEMS gyroscope performance in the presence of high power acoustic noise, 2007 IEEE International Symposium on Industrial Electronics, Vigo, Spain, (2007).

2 Dean, R., Flowers, G., Sanders, N., Horvath, R., Kranz, M., and Whitley, M. Micromachined vibration isolation filters to enhance packaging for mechanically harsh environments, Journal of Microelectronics and Electronic Packaging, 2(4), 223-231, (2005).

3 Roberts, J., Motalab, M., Hussain, S., Suhling, J. C., Jaeger, R. C., and Lall, P. Measurement of die stresses in microprocessor packaging due to thermal and power cycling, 2012 IEEE 62nd Electronic Components and Technology Conference, San Diego, California, (2012).

4 Roberts, J., Hussain, S., Rahim, M. K., Motalab, M., Suhling, J. C., Jaeger, R. C., ... Zhang, R. Characterization of microprocessor chip stress distributions during component packaging and thermal cycling. 2010 Proceedings 60th Electronic Components and Technology Conference (ECTC), Las Vegas, Nevada, (2010).

5 Zhang, S. Acoustic metamaterial design and applications, Doctoral dissertation, University of Illinois at UrbanaChampaign, (2010).

6 Yunker, W. N., Stevens, C. B., Flowers, G. T., and Dean, R. N. Sound attenuation using microelectromechanical systems fabricated acoustic metamaterials, J. Appl. Phys., 113(2), 024906, (2013).

7 Soobramaney, P. Mitigation of the effects of high levels of high-frequency noise on MEMS gyroscopes, Doctoral dissertation, Auburn University, (2013).
8 Selamet, A., Ji, Z. L., and Radavich, P. M. Acoustic attenuation performance of circular expansion chambers with offset inlet/outlet: II. comparison with experimental and computational studies, J. Sound. Vib., 213(4), 619-641, (1998).

9 Selamet, A. and Ji, Z. L. Acoustic attenuation performance of circular expansion chambers with extended inlet/outlet, J. Sound. Vib., 223(2), 197-212, (1999).

10 Selamet, A., Denia, F. D., and Besa, A. J. Acoustic behavior of circular dual-chamber mufflers. J. Sound. Vib., 265(5), 967-985, (2003).

11 Gerges, S. N. Y., Jordan, R., Thieme, F. A., Bento Coelho, J. L., and Arenas, J. P. Muffler modeling by transfer matrix method and experimental verification, J. Braz. Soc. Mech. Sci. and Eng., 27(2), 132-140, (2005).

12 Munjal, M. L. A simple numerical method for threedimensional analysis of simple expansion chamber mufflers of rectangular as well as circular cross-section with a stationary medium, J. Sound. Vib., 116(1), 71-88, (1987).

13 Kanade, S. V. and Bhattu, A. P. Optimization of sound transmission loss and prediction of insertion loss of single chamber perforated plug muffler with straight duct, $A I$ JESTEM, 6(1), 13-19, (2014).

14 Munjal, M. L. Advances in the acoustics of flow ducts and mufflers, Sadhana, 15(2), 57-72, (1990).

15 Antao, D. S., and Farouk, B. High amplitude nonlinear acoustic wave driven flow fields in cylindrical and conical resonators, J. Acoust. Soc. Am., 134(2), 917-932, (2013).

16 Friend, J. and Yeo, L. Y. Microscale acoustofluidics: microfluidics driven via acoustics and ultrasonics, Rev. Mod. Phys., 83(2), 647-704, (2011).

17 Karlsen, J. T., and Bruus, H. Forces acting on a small particle in an acoustical field in a thermoviscous fluid. Phys. Rev. E, 92(4), 043010, (2015).

18 Price, J. Acoustic waveguides, University of Colorado, Boulder, (2008).

19 Rayleigh, J. W. S. The theory of sound, MacMillan \& Co., London, (1945).

20 Munjal, M. L. Velocity ratio-cum-transfer matrix method for the evaluation of a muffler with mean flow, J. Sound. Vib., 39(1), 105-119, (1975).

21 Bejan, A. Convection heat transfer, John Wiley \& Sons, (2013). 